\title{
Special SeCtion Guest EDITORIAL
}

\section{Optical Imaging and Spectroscopy: From Benchtop to Bedside}

Following the Inter-Institute workshop on Optical Imaging from Bench to Bedside held at the National Institutes of Health in September 2000, Bruce Tromberg suggested that we publish a special section in JBO devoted to this topic, which would be sponsored by $\mathrm{NIH}$.

The timing for such an enterprise is about right. After a decade of intense efforts, a wide range of optical imaging and spectroscopic systems have been developed or are under development. These achievements have followed a quite tortuous path, requiring multi-disciplinary and multi-step approaches. At the desk, physicists and mathematicians are devising quantitative theories and developing methodologies applicable to in-vivo quantitative tissue spectroscopy and tomographic imaging in different geometries (i.e., transmission or reflection), and for different types of measurements (steadystate, frequency-resolved or time-resolved). Effects of different optical sources of contrast such as endogenous or exogenous fluorescent labels, variations in absorption (e.g., hemoglobin or chromophore concentration), and scattering are being studied. At the bench, with the help of engineers, researchers are conducting experiments on tissue-like phantoms to validate their design and methodology. However, the difficult task is to bring the imaging or spectroscopic device to the bedside. This requires fostering strong collaborations with physicians who can help to identify physiological sites where optical tech- niques may be clinically practical and can offer new diagnostic knowledge and/or less morbidity over existing methods in therapy and diagnosis. An important intermediate step has been the use of animal models for pre-clinical studies. So far, the spectroscopic power of light, along with the revolution in molecular characterization of disease processes has created a huge potential for in-vivo optical imaging and spectroscopy by adding functional information to structural characterization while avoiding ionizing radiation hazards.

Eleven papers are included in this special section. The first three papers deal with the use of fluorescence detection of disease processes with spectroscopic and imaging methods. The next five papers deal with quantitative spectroscopy, and the last three papers present imaging devices used for structural or functional characterization.

Amir H. Gandjbakhche, National Institutes of Health Michael Patterson, Hamilton Regional Cancer Center, and McMaster University

Michele Follen, M.D. Anderson Cancer Center, University of Texas

Special Section Guest Editors 